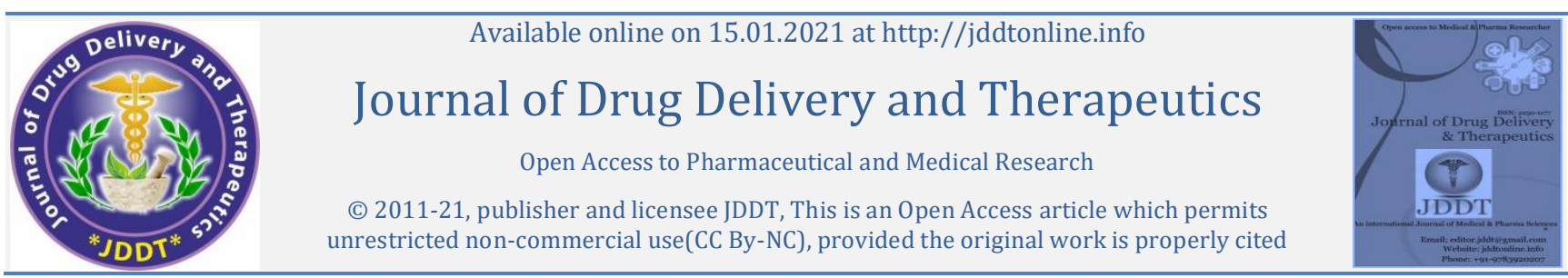

\title{
To establish bioequivalence of 50mg Metoprolol Succinate extended release tablets in normal, healthy, adult, human subject under fasting condition
}

\author{
Mahavir Singh*, Lalit Singh Ranawat \\ Geetanjali Institute of Pharmacy (Geetanjali University) Udaipur (Rajasthan), India
}

\begin{tabular}{|c|c|c|}
\hline Article Info: & & Abstract \\
\hline \multicolumn{2}{|c|}{$\begin{array}{l}\text { Cite this article as: } \\
\text { Singh M, Ranawat LS, To establish } \\
\text { bioequivalence of } 50 \mathrm{mg} \text { Metoprolol Succinate } \\
\text { extended release tablets in normal, healthy, } \\
\text { adult, human subject under fasting condition, } \\
\text { Journal of Drug Delivery and Therapeutics. } 2021 \text {; } \\
\text { 11(1): } 48-59 \\
\text { DOI: http://dx.doi.org/10.22270/jddt.v11i1.4497 }\end{array}$} & \multirow{3}{*}{ 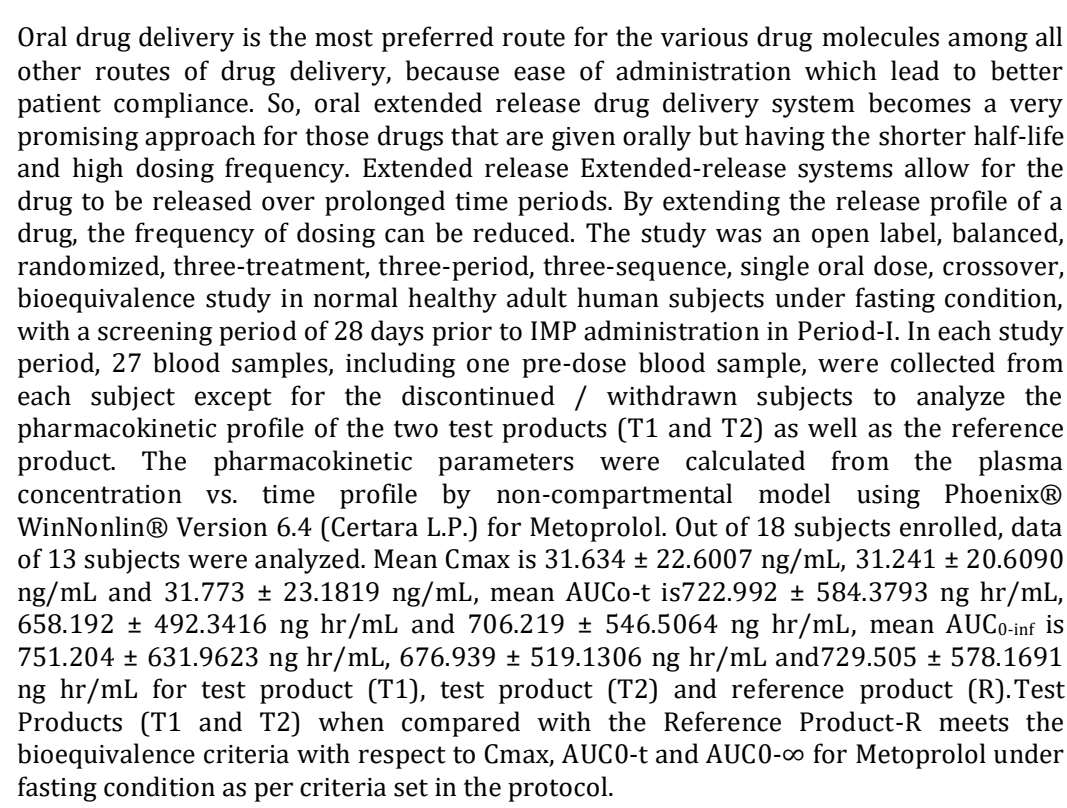 } \\
\hline India & & \\
\hline & & \\
\hline
\end{tabular}

\section{INTRODUCTION}

Recently, extended release pharmaceutical products became a very useful tool in medical practice, offering a wide range of actual and perceived advantages to the patients. Oral extended release drug delivery system becomes a very promising approach for those drugs that are given orally but having the shorter half-life and high dosing frequency. Extended release is also providing promising way to decrease the side effect of drug by preventing the fluctuation of the therapeutic concentration of the drug in the body.

There are several reasons for attractiveness of these dosage forms: provides increased bioavailability of drug product, reduction in the frequency of administration to prolong duration of effective blood levels, reduces the fluctuation of peak trough concentration and side effects and possibly improves the specific distribution of the drug ${ }^{1-4}$.

Extended release Extended-release systems allow for the drug to be released over prolonged time periods.

Sustained release: These systems maintain the rate of drug release over a sustained period.

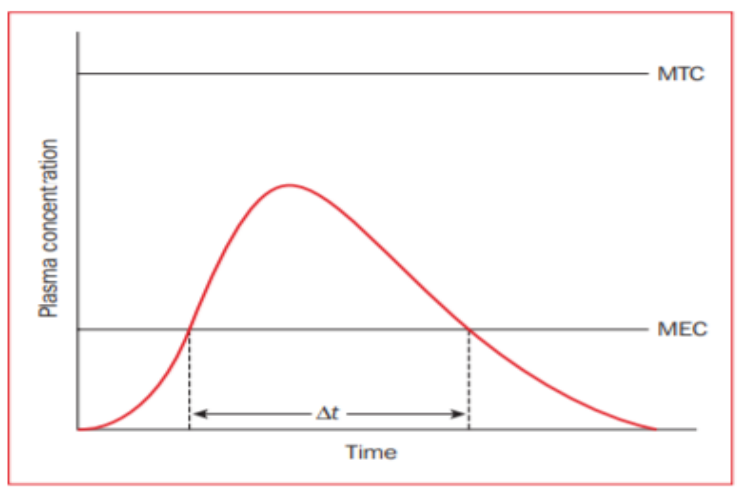

Figure 1 Idealised plasma concentration versus time profile of a sustained-release oral dosage form compared to an immediate-release dosage form.

Controlled-release: Controlled-release systems also offer a sustained-release profile but, in contrast to sustained-release forms, controlled-release systems are designed to lead to 
predictably constant plasma concentrations, independently of the biological environment of the application site.

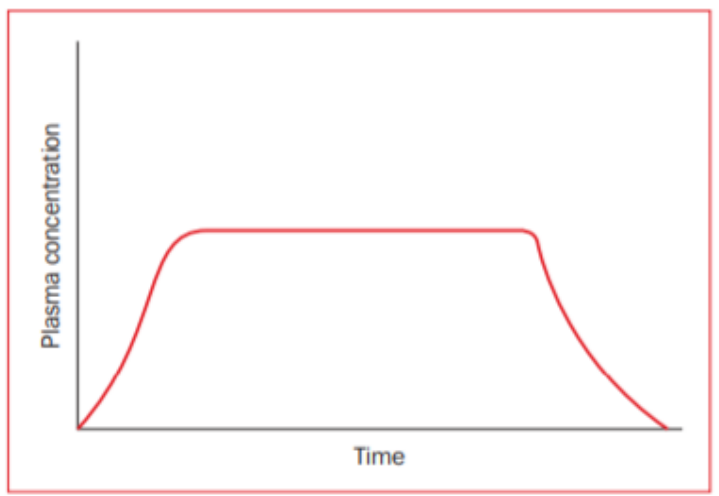

Figure 2 Idealised plasma concentrations versus time profile of a controlled-release dosage form.

\section{Rationale of Extended Drug Delivery 5}

An appropriate formulation can make the absorption, distribution, metabolism and elimination (ADME) profile of a drug much more favourable. This change of the ADME can have a profound impact on many aspects of the clinical use of the drug from patient compliance and convenience to its very efficacy, tolerance and safety parameters.

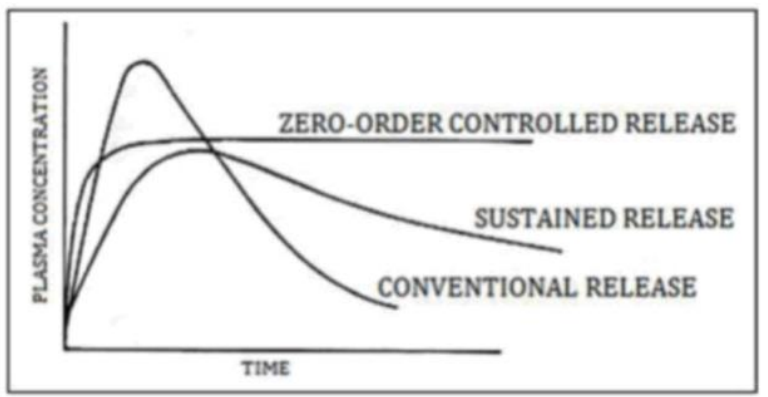

Figure 3 Plasma drug concentrations vs. time profile for oral conventional dosing and single oral dose of sustained and controlled release formulation

\section{Bioavailability:}

The rate and extent to which the active ingredient or active moiety is absorbed from a drug product and becomes available at the site of action ${ }^{6}$.

The relative amount of drug from an administered dosage form which enters the systemic circulation and the rate at which drug appears in the systemic circulation 7.

\section{Bioequivalence:}

The absence of a significant difference in the rate and extent to which the active ingredient or active moiety in pharmaceutical equivalents or pharmaceutical alternatives becomes available at the site of drug action when administered at the same molar dose under similar conditions in an appropriately designed study ${ }^{8}$.

Comparison of two medicinal products which contains the same active substance and the extent of rate of absorption of test should not differ statistically significantly to reference drug 7,9

\section{Drug profile: -}

Metoprolol is a selective $\beta 1$ receptor blocker used in treatment of several diseases of the cardiovascular system, especially hypertension 10 . The active substance Metoprolol is employed either as Metoprolol succinate or Metoprolol tartrate (where $100 \mathrm{mg}$ Metoprolol tartrate corresponds to $95 \mathrm{mg}$ Metoprolol succinate), respectively as prolongedrelease or conventional-release formulation ${ }^{11,12}$.

Dose: 25, 50, 100, $200 \mathrm{mg}$.

Absorption: completely absorbed in the gastrointestinal tract 13 .

Bioavailability: The bioavailability of a single dose is approximately $50 \%$, increasing to approximately $70 \%$ during repeated administration. The bioavailability also increases if Metoprolol is given with food.

Metabolism: Metoprolol goes through significant first-pass hepatic metabolism, mainly driven by the activity of CYP2D6 and to a lesser extent due to the activity of CYP3A4 14 .

Half-life: 3-7 hours.

Mechanism of Action: It has a relatively greater blocking effect on beta1-receptors than on beta2-receptors which are chiefly involved in Broncho and vasodilatation.

\section{MATERIALS AND METHODS:}

The study was conducted in accordance with ICH GCP guideline at Geetanjali Institute of Pharmacy, Geetanjali University, Udaipur, Rajasthan, India.

\section{Study Conduction Plan:}

This study was conducted in compliance with the protocol approved by the Independent Ethics Committee and according to Good Clinical Practice standards. No deviation from the protocol was implemented without the prior review and approval of the IEC except changes involve only administrative or logistic changes and where it might necessary to eliminate an immediate hazard to a research subject. In such case, the deviation was reported to the IEC as soon as possible.

\section{Study Population:}

Eighteen (18) Normal, Healthy, Adult, Male Human Subjects fulfilling the inclusion criteria and none of the exclusion criteria were enrolled for this study.

\section{Study Products:}

Table 1 Study Products

\begin{tabular}{|l|l|}
\hline Test Product-T1 & Metoprolol succinate Extended Release Tablets $50 \mathrm{mg}$ \\
\hline Test Product-T2 & Metoprolol succinate Extended Release Tablets $50 \mathrm{mg}$ \\
\hline Reference Product-R & TOPROL-XL@ (Metoprolol Succinate) Extended Release Tablets $50 \mathrm{mg}$ \\
\hline
\end{tabular}


The supplies were accompanied by the certificate of analysis and were conformed as to the pharmacopoeia standards. The drug content of the test product was not differing from that of the reference product by more than 5 percent. The Investigational products were stored in a restricted access area (pharmacy) in a storage cabinet maintained at a temperature specified on the label of the Investigational product. The investigational products for the test and reference formulation were retained. The retained samples were stored for a period of 5 years from the date of completion of the study.

\section{Study Design}

This study was an open label, balanced, randomized, threetreatment, three-period, three-sequence, single oral dose, crossover, bioequivalence study in normal healthy adult human subjects under fasting condition. The randomization for the study was generated using statistical software.In each period, subjects were administered either of the test or reference product, according to the randomization schedule.

\section{Study Duration}

Subjects undergo the screening procedure at least 28 days before the first day of dosing. The duration of the clinical phase was of 23 days including washout period of at least 05 days between administrations considering the half-life of the Metoprololstudy drug in each study period. This study was two period crossover study separated by 7 half-lives of washout period between the two periods.

\section{Housing and visits}

Subjects were housed in the clinical facility at least 11 hours before administration of the dose and were continued to remain in the clinical facility for at least 48 hours after the administration of investigational medicinal product in all the periods.

\section{Number of Subjects enrolled}

A sufficient number of volunteers were asked to report on the day of check-in for Period-I in order to ensure that at least 18 subjects were checked in at the beginning of the study.

A total of 20 subjects. A total of 20 subjects (Subject Nos. 1001-1018, X-1 and X-2) were checked in for Period-I of the study.

The screening phase was carried out within 28 days prior to the scheduled dosing day of Period-I. The subjects were administered the study drug in each period except for the discontinued / withdrawn subjects (Subject Nos. 1005, $1009,1011,1012,1017$ and 1018). The sequence of administration was determined by the randomization schedule. A washout period of 09 days was considered sufficient between the dosing days of any two consecutive periods. The duration of the clinical part of the study was about 22 days ( 11 hours prior to the IMP administration in Period-I until the last pharmacokinetic sample collection in Period-III).

\section{Treatments}

\section{Treatments Administered}

After an overnight fast of at least 10 hours, a single oral dose $(50 \mathrm{mg})$ of either of the test product or the reference product was administered to the subjects in sitting posture with $240 \pm 02 \mathrm{~mL}$ of drinking water at ambient temperature as per randomization schedule.

\section{Table 2 Treatment sequence}

\begin{tabular}{|l|l|l|l|}
\hline & Period-I & Period-II & Period-III \\
\hline \multirow{3}{*}{ Sequence } & Treatment-T1 (Test) & Treatment-T2 (Test) & Treatment-R (Reference) \\
\cline { 2 - 4 } & Treatment-R (Reference) & Treatment-T1 (Test) & Treatment-T2 (Test) \\
\cline { 2 - 4 } & Treatment-T2 (Test) & Treatment-R (Reference) & Treatment-T1 (Test) \\
\hline
\end{tabular}

The IMP administration was as per the randomization schedule and under open label conditions.

The tablet was swallowed whole without chewing or crushing.

The time of administration of the investigational medicinal product was the time at which the subject completed drinking $240 \pm 02 \mathrm{ml}$. This activity was followed by a mouth check to assess the compliance to dosing. The subjects remained in supine or semi-recumbent positions after dosing and up to first 08 hours post-dose in each period. They rose up only with assistance during that period of time. They were allowed to sit in the bed at the time of taking the meal and it was not considered as the protocol deviation. Thereafter, they were allowed to engage only in normal activities while avoiding severe physical exertion. All the planned activities till 08 hours post-dose including the allowed time window to perform such activities were carried out in supine or semi-recumbent position at bed side.

\section{Method of Assigning Subjects to Treatment Groups}

This was a randomized study design. The order of receiving Test Products (T1 and T2) and Reference Product-R for each subject in each period of the study was determined according to the randomization schedule. Equal allocation of subjects to each sequence was ensured. The study personnel involved in the sample analysis were kept blinded from the randomization code during the entire study. Subjects were sequentially assigned a subject number as per the Arrival Sequence Number, which was allotted on the basis of the subject's reporting time to the facility on the day of check-in for Period-I and compliance to the requirements of the protocol.

\section{Selection of Dose in the Study}

Metoprolol $50 \mathrm{mg}$ can be safely administered for various indications. The primary objective of the study was to assess the bioequivalence of the test products (T1 and T2) in comparison to the reference product. Hence, as per the sponsor's recommendation, the aforementioned dose was selected for the study.

\section{Washout Period}

The washout period was of at least 05 days between dosing days of any two consecutive periods. 


\section{Selection and Timing of Dose for Each Subject}

A total of 27 blood samples, (each sample of $05 \mathrm{~mL}$ ), will be collected from each subject. The venous blood samples will be withdrawn at the predetermined time interval.

\section{Dosing Procedures and Compliance Assessment}

\section{Duration of Fasting and Distribution of Meals}

All subjects were required to fast overnight for at least 10 hours prior to dosing and 04 hours post dose in each period. Lunch was provided after 04 hours of dose administration.

Standardized meal was served to the subjects at appropriate times during their stay in the clinical facility. The contents of the meals served during each period at various time points were identical. The subjects received lunch at least $04 \mathrm{hrs}$ after dosing and further meals were served at appropriate intervals from then on, until checkout.

Subjects will refrain from drinking water from 1 hour before till 1 hour after each dosing in each period except for the 240 $\pm 02 \mathrm{~mL}$ of water administered during dosing. Prior to and thereafter, water shall be consumed as required.

The details regarding the total number of samples collected during the study is given in the below table:

Table 3 List of samples collected

\begin{tabular}{|l|l|l|l|}
\hline S. N. & Subject No. & $\begin{array}{l}\text { Total No. of Samples } \\
\text { Collected }\end{array}$ & Remarks \\
\hline 1. & $\begin{array}{l}1001-1004,1006-1008, \\
1010 \text { and } 1014-1016\end{array}$ & $891(81 \times 11)$ & All the samples were collected. \\
\hline 2. & 1005 & $21(21 \times 01)$ & $\begin{array}{l}\text { The subject was withdrawn from the study on medical grounds in } \\
\text { Period-I. }\end{array}$ \\
\hline 3. & 1009 & $27(27 \times 01)$ & $\begin{array}{l}\text { The subject discontinued from the study on his own accord in } \\
\text { Period-II. }\end{array}$ \\
\hline 4. & 1011 & $54(54 \times 01)$ & The subject was withdrawn from Period- II on medical grounds. \\
\hline 5. & 1012 & $27(27 \times 01)$ & The subject discontinued from Period-II and III on his own accord. \\
\hline 6. & 1013 & $67(67 \times 01)$ & The subject was withdrawn from Period- III on medical grounds. \\
\hline 7. & 1017 & $27(27 \times 01)$ & The subject discontinued from Period-II and III on his own accord. \\
\hline 8. & 1018 & $55(55 \times 01)$ & The subject was withdrawn from Period- III on medical grounds. \\
\hline
\end{tabular}

Total No. of samples to be collected as per the protocol: 1458

Total No. of samples collected during the study: 1169

\section{Dosage Administration:}

After an overnight fast of at least 10 hours, a single oral dose $(50 \mathrm{mg}$ ) of either of the test product or the reference product was administered to the subjects in sitting posture with $240 \pm 02 \mathrm{~mL}$ of drinking water at ambient temperature. The IMP administration was as per the randomization schedule and under open label conditions.

The time of administration of the investigational medicinal product was the time at which the subject completed drinking $240 \pm 02 \mathrm{~mL}$ of water.

Compliance for dosing was assessed by a thorough check of the oral cavity by using a disposable tongue depressor and torch immediately after dosing by trained personnel in guidance with medical officer. The tablet was instructed to be swallowed as whole without chewing or crushing Record of dosing for individual subject was maintained in Case Report form. Dosing was carried out in the morning after an overnight fast of at least 10 hours.

\section{Selection and timing of dose for each subject}

As per the protocol, a total of twenty-seven (27) blood samples, each of $05 \mathrm{~mL}$ were to be collected from each subject in each period.

The venous blood samples were withdrawn at pre-dose (0.000 hour) and at 1.000, 2.000, 3.000, 4.000, 5.000, 6.000, $7.000,8.000,9.000,10.000,11.000,12.000,13.000,14.000$, $15.000,16.000,18.000,20.000,22.000,24.000,26.000$, $30.000,34.000,38.000,42.000$ and 48.000 hours.

\section{Sample Handling and Processing:}

\section{Blood Sampling and Processing}

Blood Samples Collection: -Blood samples were collected through an indwelling intravenous cannula (Venflon) placed in a forearm vein of the subjects. Cannula was removed after collection of 24.000 hours post-dose sample. Blood samples at and after 26.000-hour post-dose were collected through via fresh vein puncture. Immediately after collection of blood, the collection tube was inverted gently several times to ensure the mixing of tube contents (i.e. anticoagulant).

\section{Sample Analysis}

\section{Plasma Separation}

The blood samples were centrifuged at $3000 \mathrm{rpm}$ for 05 minutes at $4^{\circ} \mathrm{C}$ to separate plasma. For precaution purpose, the blood samples were kept in ice cold water bath before centrifugation and during separation. The separated plasma was transferred to pre-labelled polypropylene tubes in two aliquots (around $0.7 \mathrm{~mL}$ in first aliquot and remaining volume in second aliquot) and the samples were stored upright in a freezer at a temperature $-65 \pm 10^{\circ} \mathrm{C}$ for interim storage till transfer of the same to the bio-analytical department. During transfer, the samples were kept in a box containing adequate amount of dry ice with data logger.

\section{Bioanalytical Procedures}

The plasma samples of subjects were analyzed using a validated LC-MS/MS method for Metoprolol at the 
Bioanalytical facility of Lambda Therapeutic Research Ltd., Ahmedabad, India. The analysis was conducted as per the Method SOP. Calibration curves using 8-point calibration curve standards, were used to determine the concentrations of Metoprolol in the samples of all analyzed subjects.

\section{Analytical run organization for study sample analysis}

Subject samples were processed using liquid-liquid extraction method. Subject samples were processed with calibration curve standards, interspersed quality control samples and subject samples with all time-points of a particular period of each subject positioned as consecutive samples.

In all the cases, analytical run was rejected or accepted on the basis of the results obtained for quality control samples and calibration curve standards run with that particular analytical run.

\section{Adverse Event Reporting:}

All adverse events that were reported properly on the adverse event form or serious adverse event reporting form depending on the type of the event. In particular the information was included description of the event, details of the occurrence, frequency of adverse event, description of the severity of the event, any treatment or diagnostic steps taken in relation to the event, description of the outcome of the event, judgment by the medical officer of any relationship of the event to study medication or procedures.

All adverse events and serious adverse events whether drug related or not were reported to the sponsor and IEC by Investigator(s), in accordance with applicable regulatory guideline.

ASerious Adverse Event was any untoward medical occurrence at any dose that results in any of the following outcome:

$>$ Death

$>$ Life-threatening (i.e. the subject was at immediate risk of death as the event actually occurred, not as it had occurred in a more serious form)

> Requires hospitalisation or prolongation of existing hospitalisation

> Persistent or significant disability / incapacity

$>$ Congenital anomaly/birth defect

\section{Statistical Methods}

\section{Statistical and Analytical Plans}

Descriptive statistics were to be calculated and reported for all pharmacokinetic parameters for Metoprolol.

The ln-transformed pharmacokinetic parameters Cmax, AUC0-t and AUC0- $\infty$ were to be subjected to Analysis of Variance (ANOVA) for Metoprolol.

ANOVA model was to be included Sequence, Formulation and Period as fixed effects and Subject (Sequence) as a random effect. Sequence effect was to be tested using Subject (Sequence) as an error term. Each analysis of variance was to be included calculation of least-squares means, the difference between the adjusted formulation means and the standard error associated with the differences.

An F-test was to be performed to determine the statistical significance of the effects involved in the model at a significance level of 5\% (alpha $=0.05)$.

The power of the study was to be computed and reported for In-transformed pharmacokinetic parameters Cmax, AUC0-t and AUC0- $\infty$ for Metoprolol.

Ratio of geometric least squares means of test and reference formulations was to be computed and reported for lntransformed pharmacokinetic parameters Cmax, AUC0-t and AUC0- $\infty$ for Metoprolol.

Inter and Intra-subject variability was to be computed and reported for ln-transformed pharmacokinetic parameters Cmax, AUC0-t and AUC0- $\infty$ for Metoprolol.

Any missing samples (M) or non-reportable (NR) concentration values were to be disregarded in pharmacokinetic and statistical analysis.

Using two one-sided tests for bioequivalence, $90 \%$ confidence intervals for the ratio of geometric least squares means between drug formulations were to be calculated for In-transformed data of Cmax, AUC0-t and AUC0- $\infty$ for Metoprolol.

Criteria for conclusion of bioequivalence were as follows:

Bioequivalence of the two test products (T1 and T2) with that of the reference product was to be concluded, if the $90 \%$ confidence interval fell within the acceptance range as defined below for ln-transformed pharmacokinetic parameters for Metoprolol.

Parameters

Acceptance Range of $90 \%$ CI

Cmax, AUC0-t and AUC0- $\infty \quad 80.00-125.00 \%$

All statistical analyses for Metoprolol were to be performed using PROC MIXED of SAS® Version 9.4 (SAS Institute Inc., USA).

\section{RESULTS:}

\section{Pharmacokinetic and Statistical Evaluation \\ Data Sets Analyzed}

Total 13 subjects were included in the pharmacokinetic and statistical analysis.

\section{Demographic and Other Baseline Characteristics}

Normal, Healthy, Adult, Male Human subjects between 18-45 years (both inclusive) of age were screened for Inclusion /Exclusion criteria as mentioned in the Study Protocol.

Table 4 Demographic profile of subjects enrolled in the BE Study

\begin{tabular}{|l|l|l|l|l|}
\hline \multicolumn{5}{|c|}{$\mathbf{N}=\mathbf{1 8}$ (Subjects who were dosed in the study) } \\
\hline Parameters & Age (years) & Height $\mathbf{( c m )}$ & Weight $\mathbf{( k g )}$ & BMI Value $\mathbf{( k g / \mathbf { m } ^ { 2 } )}$ \\
\hline Mean & 27.8 & 164.47 & 58.938 & 21.761 \\
\hline $\mathbf{I S D}$ & 6.21 & 6.237 & 8.4410 & 2.3800 \\
\hline
\end{tabular}


Table 5 Demographic profile of subjects included in the final statistical analysis

\begin{tabular}{|c|c|c|c|c|}
\hline \multicolumn{5}{|c|}{ N = 13 (Subjects included in BE evaluation) } \\
\hline Parameters & Age (yr.) & Height (cm) & Weight (kg) & BMI Value $\left(\mathrm{kg} / \mathrm{m}^{2}\right)$ \\
\hline Mean & 29.1 & 164.96 & 59.938 & 21.953 \\
\hline \pm SD & 6.26 & 6.744 & 8.6575 & 2.0586 \\
\hline
\end{tabular}

\section{Measurement of Treatment Compliance}

Compliance for dosing was assessed by monitoring the subject till they swallow tablet and then a thorough check of the oral cavity was done by the study personnel using a torch. The duplicate label of dispensed container was then pasted on the 'Dosing' section of individual Case Report Form (CRF). It was further confirmed by the measurement of plasma levels of the drug taken in the study.

Blood time points were considered for statistical analysis.

All missing samples were not included for pharmacokinetic and statistical analysis.

\section{Safety Evaluation}

\section{Extent of Exposure:}

\section{Table 6 Subject Exposure}

\begin{tabular}{|l|l|l|}
\hline Test Product-T1 & Test Product-T2 & Reference Product-R \\
\hline 14 & 16 & 14 \\
\hline One & One & One \\
\hline
\end{tabular}

A total of 20 subjects were checked in for the study. Out of these 20subjects, 18 subjects were dosed in Period-I of the study. The safety assessment includes information for all 18 subjects who were dosed at least once during this study.

\section{Adverse Events}

Four (04) significant adverse events (AEs) were reported by four (04) subjects during the conduct of the study. Two (02) AEs were reported in Period-I and two (02) AEs were reported in Period-III of the study. Two (02) AEs were reported in the subjects after administration of Test Product$\mathrm{T} 1$, one (01) $\mathrm{AE}$ was reported in the subject after administration of Test Product-T2 and one (01) AE was reported in the subject after administration of Reference Product-R. All the AEs were mild in nature. The causality assessment was judged as unlikely for three (03) AEs and as possible for one (01) AE. The subjects were withdrawn on medical grounds. They were treated appropriately and followed up until resolution of their AEs.

\section{Analysis of adverse event}

The adverse event reported during the study was analyzed for onset, relationship, likelihood, severity, seriousness, duration etc. The adverse event was not life threatening or required the subject to be hospitalized.

Table 7 Analysis of adverse events

\begin{tabular}{|l|l|l|}
\hline \multirow{2}{*}{ Body System/ Adverse Event } & Reported Incidence by Treatment Group \\
\cline { 2 - 3 } & Test & Reference \\
\hline \multicolumn{3}{|c|}{ Skin and subcutaneous tissue disorder } \\
\hline Pruritus generalized & $0(00.00 \%)$ & $01(7.14 \%)$ \\
\hline \multicolumn{1}{|c|}{ Musculoskeletal and connective tissue disorders } \\
\hline Musculoskeletal chest pain & $01(7.14 \%)$ & $01(7.14 \%)$ \\
\hline \multicolumn{1}{|c|}{ Electrocardiogram } \\
\hline Electrocardiogram General disorders and administration site conditions \\
\hline \multicolumn{2}{|c|}{$01(7.14 \%)$} & $0(00.00 \%)$ \\
\hline Pyrexia & $0(00.00 \%)$ & $01(6.25 \%)$ \\
\hline
\end{tabular}

There were no deaths or significant adverse events during the conduct of this study.

\section{Plasma Concentration Profile:}

The measured individual and mean plasma concentrations of Metoprolol for both the test products and reference product, for each subject for each time point were calculated.
Individual and mean plasma concentrations of 13 subjects (who complete the both the clinical phase of the study successfully) utilized for Pharmacokinetic and Statistical analysis at different sampling time points of Reference product (R) and Test product (T1) \& Test product (T2). 
Table 8 Mean Plasma Concentrations $(\mathrm{pg} / \mathrm{ml})$ for Test and Reference formulation.

\begin{tabular}{|c|c|c|c|}
\hline PK Parameter & $\begin{array}{c}\text { C max (Test 1) } \\
(\mathrm{ng} / \mathrm{mL})\end{array}$ & $\begin{array}{c}\text { C max (Test 2) } \\
(\mathrm{ng} / \mathrm{mL})\end{array}$ & $\begin{array}{c}\text { C max (Reference R) } \\
(\mathrm{ng} / \mathrm{mL})\end{array}$ \\
\hline $\mathbf{N}$ & 13 & 11 & 13 \\
\hline MIN & 10.425 & 10.472 & 9.759 \\
\hline MAX & 76.008 & 73.019 & 76.402 \\
\hline MEAN & 31.634 & 31.241 & 31.773 \\
\hline SD & 22.6007 & 20.6090 & 23.1819 \\
\hline MEDIAN & 22.613 & 22.154 & 21.636 \\
\hline GEOMETRIC MEAN & 25.873 & 26.681 & 25.807 \\
\hline$\% \mathrm{CV}$ & 71.4 & 66.0 & 73.0 \\
\hline
\end{tabular}

\section{Graphs:}

The untransformed and Log -transformed Mean Plasma Concentrations at each time points are as presented below: Mean Plasma Concentration Graphs

Linear Plot

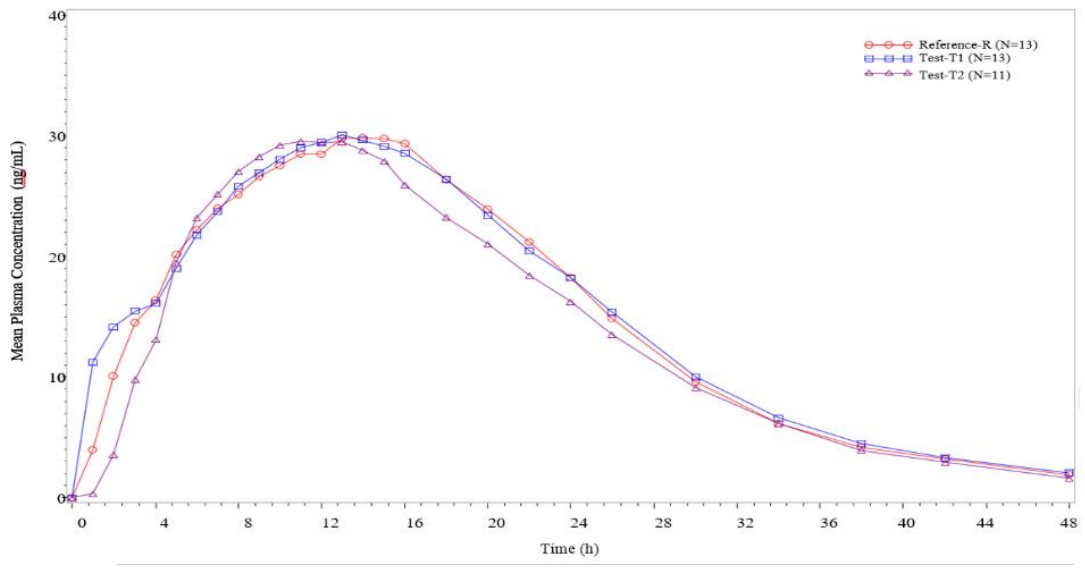

\section{Linear Plot}

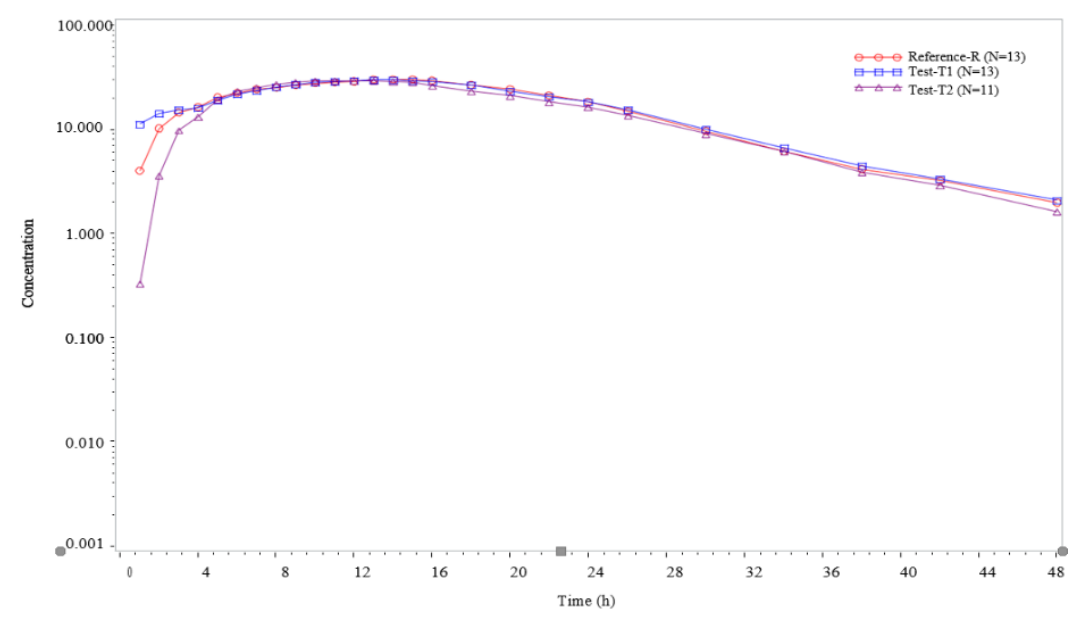

Figure 4 Summary plot of individual plasma concentration vs. time curve for Metoprolol 


\section{Linear Plot (Reference Product)}

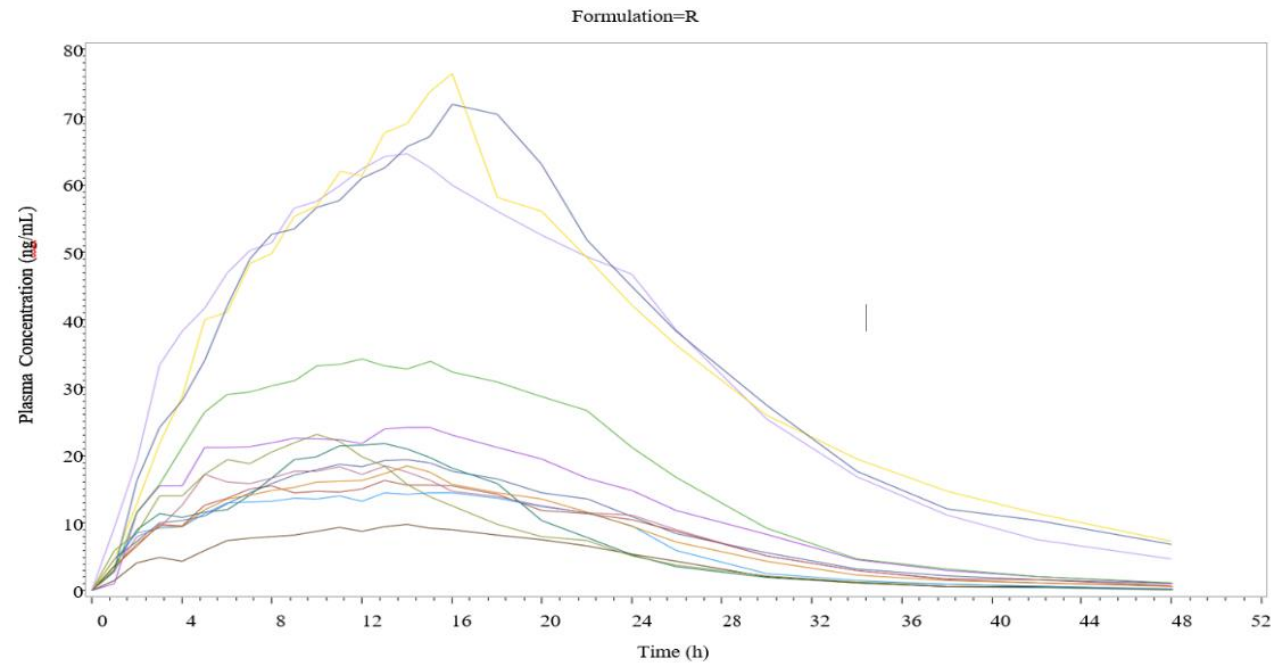

Semi-log Plot (Reference Product)

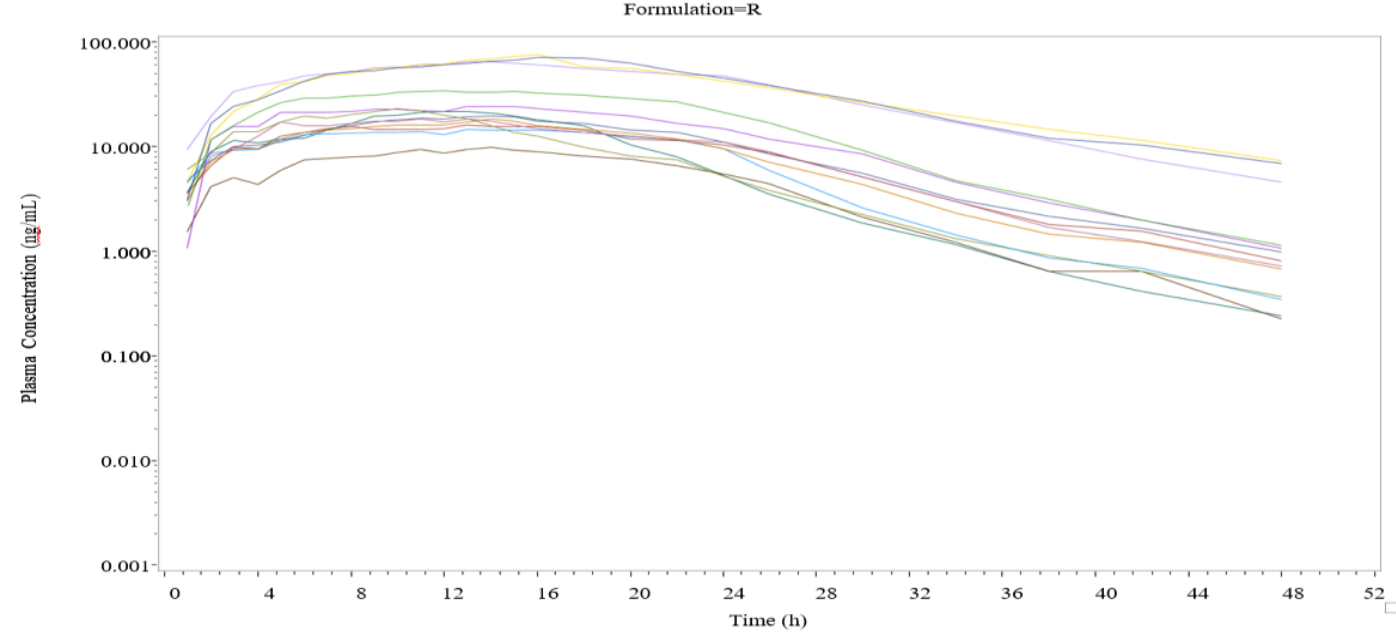

Linear Plot (Test Product-T1)

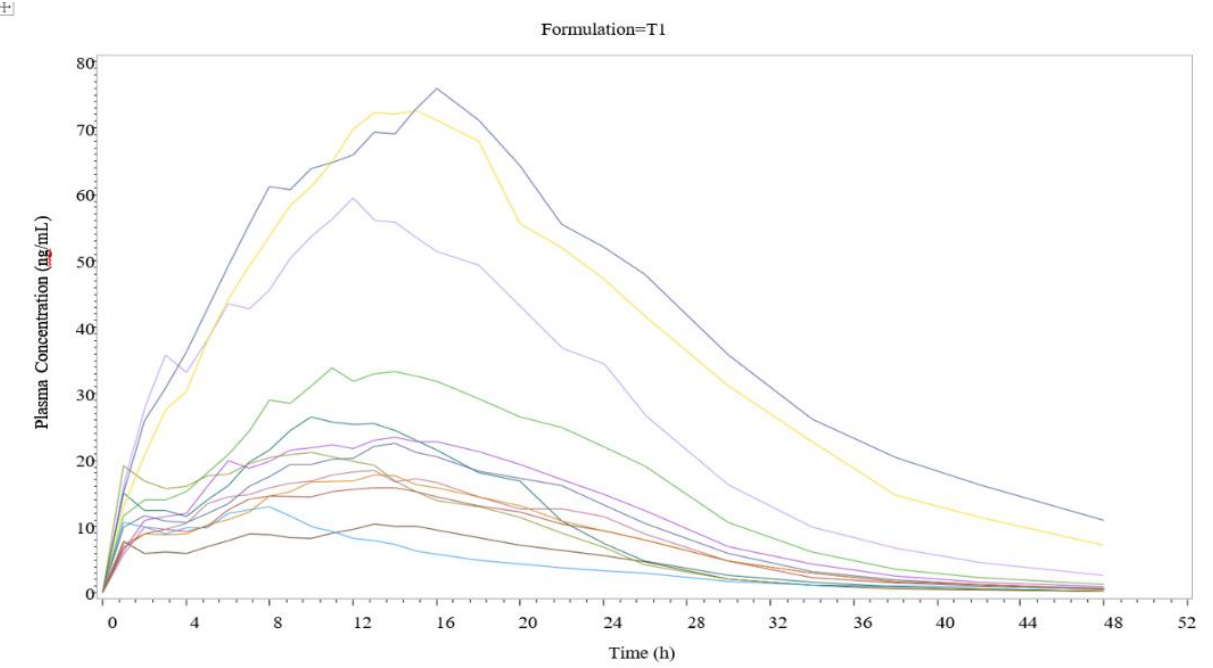

Semi-log Plot (Test Product-T1) 


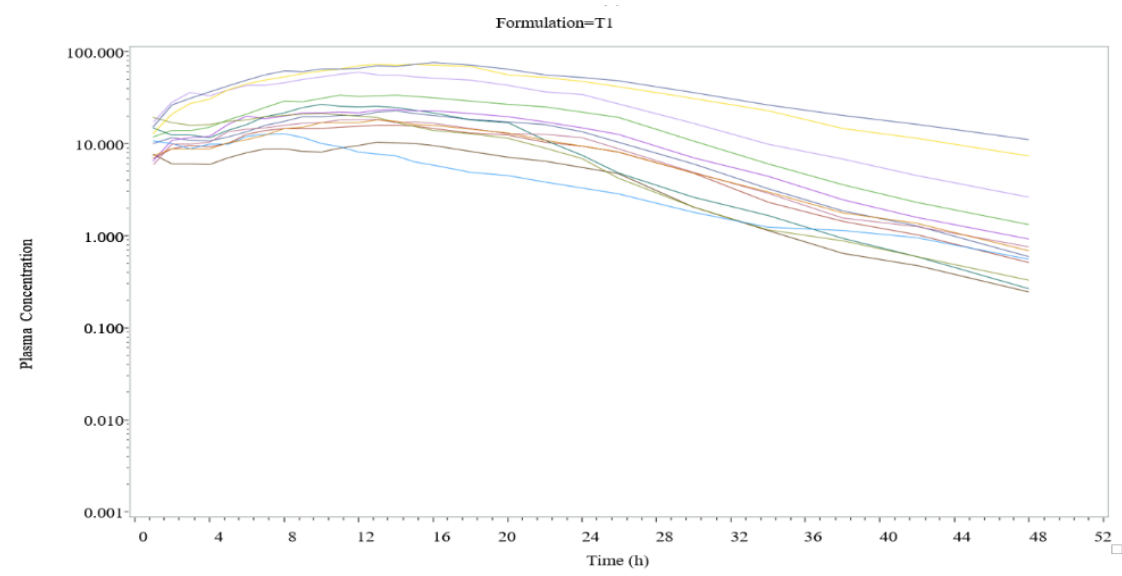

Linear Plot (Test Product-T2)

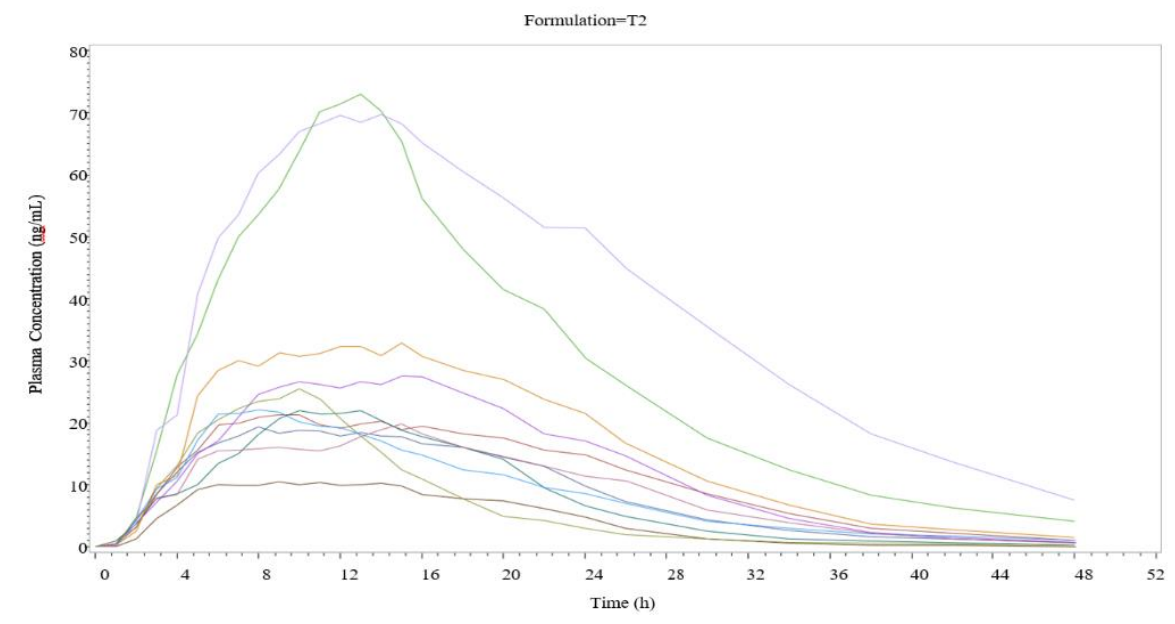

\section{Semi-log Plot (Test Product-T2)}



Figure 5 Mean Plasma Concentrations vs. Time Curve and Mean Plasma Concentration vs. Time Curve onLogarithmic Scale

\section{Pharmacokinetic Parameters:}

The pharmacokinetic parameters were calculated from the plasma concentration vs. time profile by non-compartmental model using Phoenix $\AA$ WinNonlin ${ }^{\circledR}$ Version 6.4 (Certara L.P.) for Metoprolol. Statistical comparison of the pharmacokinetic parameters of the three formulations (two tests and one reference formulations) was carried out using PROC MIXED of SAS® Version 9.4 (SAS Institute Inc., USA) to assess the bioequivalence between two tests and one reference formulations.

Based on the data analyzed from 13 subjects for Metoprolol the pharmacokinetics was assessed for the comparison of
Test Product-T1 vs. Reference Product-R and Test ProductT2 vs. Reference Product-R.

The tests (T1 and T2) to reference (R) ratio of geometric least square means with corresponding 90\% CI for lntransformed pharmacokinetic parameter Cmax, AUC0-t and AUC $0-\infty$ were within the acceptance range of 80.00$125.00 \%$.

The Mean \pm SD pharmacokinetic parameters estimated for the Test products $\mathrm{T} 1 \& \mathrm{~T} 2$ and Reference product (R) formulations of Metoprolol for $\mathrm{N}=13$ are as follows: 
Table 9 Mean PK Parameters

\begin{tabular}{|c|c|c|c|c|}
\hline \multicolumn{5}{|c|}{ Test Formulation (T1) } \\
\hline Measures & $\operatorname{Tmax}(\mathrm{h})$ & $\mathrm{Cmax}(\mathrm{ng} / \mathrm{mL})$ & AUC0-t (ng.h/mL) & AUCO-inf (ng.h/mL) \\
\hline $\mathbf{N}$ & 13 & 13 & 13 & 13 \\
\hline Mean & 12.540 & 31.634 & 722.992 & 751.204 \\
\hline SD & 2.2560 & 22.6007 & 584.3793 & 631.9623 \\
\hline CV (\%) & 18.0 & 71.4 & 80.8 & 84.1 \\
\hline Geometric Mean & 12.335 & 25.873 & 562.964 & 576.367 \\
\hline \multicolumn{5}{|c|}{ Test Formulation (T2) } \\
\hline Measures & $\mathrm{Tmax}(\mathrm{h})$ & Cmax (ng/mL) & AUC0-t (ng.h/mL) & AUCO-inf (ng.h/mL) \\
\hline $\mathbf{N}$ & 11 & 11 & 11 & 11 \\
\hline Mean & 11.820 & 31.241 & 658.192 & 676.939 \\
\hline SD & 2.8549 & 20.6090 & 492.3416 & 519.1306 \\
\hline CV (\%) & 24.2 & 66.0 & 74.8 & 76.7 \\
\hline Geometric Mean & 11.490 & 26.681 & 539.661 & 550.608 \\
\hline \multicolumn{5}{|c|}{ Reference Formulation (R) } \\
\hline Measures & $\operatorname{Tmax}(\mathrm{h})$ & Cmax (ng/mL) & AUC0-t (ng.h/mL) & AUCO-inf (ng.h/mL) \\
\hline $\mathbf{N}$ & 13 & 13 & 13 & 13 \\
\hline Mean & 13.619 & 31.773 & 706.219 & 729.505 \\
\hline SD & 1.6112 & 23.1819 & 546.5064 & 578.1691 \\
\hline CV (\%) & 11.8 & 73.0 & 77.4 & 79.3 \\
\hline Geometric Mean & 13.526 & 25.807 & 558.692 & 571.217 \\
\hline
\end{tabular}

\section{Relative Bioavailability Results for Metoprolol}

The relative bioavailability analyses (i.e. geometric least squares mean, ratio, 90\% confidence interval, intra subject
$\mathrm{CV}$ and power) of Test Product-T1 vs. Reference Product-R and Test Product-T2 vs. Reference Product-R for Metoprolol are summarized in the following table:

Table 10 Relative Bioavailability Results

\begin{tabular}{|c|c|c|c|c|c|c|}
\hline \multicolumn{6}{|l|}{ T1 vs. $R$} & \multirow[b]{2}{*}{$\begin{array}{l}\text { Power } \\
(\%)\end{array}$} \\
\hline Parameters & $\begin{array}{l}\text { Test Product } \\
\text { (T1) }\end{array}$ & $\begin{array}{l}\text { Reference } \\
\text { Product (R) }\end{array}$ & $\begin{array}{l}\text { Ratio (T1/R) } \\
\%\end{array}$ & $\begin{array}{l}90 \% \text { Confidence } \\
\text { interval }\end{array}$ & $\begin{array}{l}\text { Intra } \\
\text { subject CV } \\
(\%)\end{array}$ & \\
\hline $\operatorname{lnCmax}$ & 26.279 & 25.617 & 102.6 & $95.63-110.04$ & 9.9 & 99.9 \\
\hline $\ln A U C 0-t$ & 574.371 & 555.916 & 103.3 & $94.04-113.52$ & 13.4 & 98.6 \\
\hline $\ln A U C 0-\infty$ & 587.361 & 567.737 & 103.5 & $94.19-113.63$ & 13.3 & 98.6 \\
\hline \multicolumn{7}{|l|}{ T2 vs. $R$} \\
\hline \multirow{2}{*}{ Parameters } & \multicolumn{3}{|c|}{ Geometric Least Squares Means } & \multirow{2}{*}{$\begin{array}{l}90 \% \text { Confidence } \\
\text { interval }\end{array}$} & \multirow{2}{*}{$\begin{array}{l}\text { Intra } \\
\text { subject CV } \\
(\%)\end{array}$} & \multirow{2}{*}{$\begin{array}{l}\text { Power } \\
(\%)\end{array}$} \\
\hline & $\begin{array}{l}\text { Test Product } \\
\text { (T2) }\end{array}$ & $\begin{array}{l}\text { Reference } \\
\text { Product (R) }\end{array}$ & $\begin{array}{l}\text { Ratio }(\mathrm{T} 2 / \mathrm{R}) \\
\%\end{array}$ & & & \\
\hline $\operatorname{lnCmax}$ & 28.134 & 25.617 & 109.8 & $102.04-118.20$ & 9.9 & 99.9 \\
\hline $\ln A U C 0-t$ & 584.411 & 555.916 & 105.1 & $95.26-116.01$ & 13.4 & 97.9 \\
\hline $\ln A U C 0-\infty$ & 597.239 & 567.737 & 105.2 & $95.36-116.05$ & 13.3 & 98.0 \\
\hline
\end{tabular}


Peak Plasma Concentration $\left(\mathrm{C}_{\max }\right)$ and Time to reach Peak Concentration $\left(T_{\max }\right)$ :

In Metoprolol, the arithmetic means for un-transformed Cmax were $31.634 \pm 22.6007 \mathrm{ng} / \mathrm{mL} \& 31.241 \pm 20.6090$ $\mathrm{ng} / \mathrm{mL}$ for Test Products (T1 \& T2) and $31.773 \pm 23.1819$ $\mathrm{ng} / \mathrm{mL}$ for Reference Product $\mathrm{R}$, respectively.

\section{Area under the Plasma Concentration-Time Curve (AUC ${ }_{0-t}$ and $\mathrm{AUC}_{0 \text {-inf): }}$}

In Metoprolol, the arithmetic means for un-transformed AUC0-t were $722.992 \pm 584.3793$

ng.hr/mL \&658.192 \pm 492.3416 for Test Product (T1) \& Test Product (T2) and 706.219 \pm 546.5064 ng.hr $/ \mathrm{mL}$ for Reference Product (R), respectively.
In Metoprolol, the arithmetic means for un-transformed AUC0- $\infty$ were $751.204 \pm 631.9623 \mathrm{ng} . \mathrm{hr} / \mathrm{mL} \& 676.939 \pm$ 519.1306 for Test Product (T1) \& Test Product (T2) and 14466.263 ng.hr/mL for Reference Product B, respectively.

Elimination Rate Constant $\left(\mathrm{K}_{\mathrm{el}}\right)$ and Elimination HalfLife $\left(t_{1} \frac{1}{2}\right)$ :

In Metoprolol, the arithmetic mean Elimination Rate Constant (Kel) were $0.099 \pm 0.0200 \mathrm{hr}$. \& $0.103 \pm 0.0290 \mathrm{hr}$. for Test Products (T1 \& T2) and $0.097 \pm 0.0152 \mathrm{hr}$. for Reference Product R.

In Metoprolol, the arithmetic mean terminal half-life was $7.332 \pm 1.6973 \mathrm{hr}$. \&7.157 $\pm 1.8584 \mathrm{hr}$. for Test Products (T1 $\& \mathrm{~T} 2$ ) and $7.286 \pm 1.1902$ for Reference (Product R).

Table 11 Pharmacokinetic Summary Results

\begin{tabular}{|c|c|c|c|}
\hline \multirow{2}{*}{ Parameters (Units) } & \multicolumn{3}{|c|}{$\begin{array}{c}\text { Mean } \pm \text { SD } \\
\text { (untransformed data) }\end{array}$} \\
\hline & $\begin{array}{l}\text { Test Product-T1 } \\
(\mathrm{N}=13)\end{array}$ & $\begin{array}{l}\text { Test Product-T2 } \\
(\mathrm{N}=11)\end{array}$ & $\begin{array}{l}\text { Reference Product-R } \\
(\mathrm{N}=13)\end{array}$ \\
\hline $\operatorname{Tmax}(\mathrm{h})$ & $13.000(8.017-16.000)$ & $13.000(8.000-15.000)$ & $14.000(10.000-16.017)$ \\
\hline $\mathrm{Cmax}(\mathrm{ng} / \mathrm{mL})$ & $31.634 \pm 22.6007$ & $31.241 \pm 20.6090$ & $31.773 \pm 23.1819$ \\
\hline AUC0-t (ng.h/mL) & $722.992 \pm 584.3793$ & $658.192 \pm 492.3416$ & $706.219 \pm 546.5064$ \\
\hline 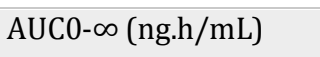 & $751.204 \pm 631.9623$ & $676.939 \pm 519.1306$ & $729.505 \pm 578.1691$ \\
\hline$\lambda z(1 / h)$ & $0.099 \pm 0.0200$ & $0.103 \pm 0.0290$ & $0.097 \pm 0.0152$ \\
\hline$t^{1} 1 / 2(h)$ & $7.332 \pm 1.6973$ & $7.157 \pm 1.8584$ & $7.286 \pm 1.1902$ \\
\hline AUC_\%Extrap_obs (\%) & $2.302 \pm 2.1919$ & $1.980 \pm 1.2977$ & $2.180 \pm 1.5954$ \\
\hline $\mathrm{R} 2$ adjusted & $0.995 \pm 0.0052$ & $0.993 \pm 0.0053$ & $0.992 \pm 0.0071$ \\
\hline Tlag (h) & $0.000(0.000-0.000)$ & $0.000(0.000-1.033)$ & $0.000(0.000-0.000)$ \\
\hline
\end{tabular}

\section{ANOVA:}

Table 12 ANOVA Calculation

\begin{tabular}{|l|l|l|l|l|}
\hline Parameters & \multicolumn{2}{c}{ ANOVA (p-value) } & CV (\%) \\
\hline InCmax & Formulation & Sequence & Period & 77.3 \\
\hline InAUC0-t & 0.1051 & 0.9715 & 0.0859 & 86.1 \\
\hline InAUC0- $\infty$ & 0.6722 & 0.9967 & 0.3095 & 88.7 \\
\hline
\end{tabular}

\section{ANOVA Summary:}

The Analysis of Variance (ANOVA) was carried out on logtransformed pharmacokinetic parameter $\mathrm{C}_{\max }, \mathrm{AUC}_{0-\mathrm{t}}$ and


Institute Inc., USA).

The ln-transformed pharmacokinetic parameters Cmax, AUC0-t and AUCO- $\infty$ were to be subjected to Analysis of Variance (ANOVA).
The power of the study was to be computed and reported for ln-transformed pharmacokinetic parameters Cmax, AUC0-t and AUC0- $\infty$ for Metoprolol.

In analysis of Metoprolol, there was no significant sequence, period and treatment effect was observed for logtransformed pharmacokinetic parameter $\mathrm{C}_{\max }, \mathrm{AUC}_{0-\mathrm{t}}$ and AUC $_{0 \text {-inf. }}$ 
Table 13 Intra-Subject Variability of Log transformed Pk Parameters Test-T1 Vs Reference-R

\begin{tabular}{|l|l|l|l|}
\hline Measures & \multicolumn{2}{l|}{ AUC0-t (ng.h/mL) } & AUC0-inf (ng.h/mL) \\
\hline $\mathbf{9 0 \%}$ Confidence Interval (T1 Vs. R) & & \\
\hline In-transformed Lower & 95.63 & 94.04 & 94.19 \\
\hline Upper & 110.04 & 113.52 & 113.63 \\
\hline Power (\%) & 99.9 & 98.6 & 98.6 \\
\hline Bioequivalence & YES & YES & YES \\
\hline
\end{tabular}

Table 14 Intra-Subject Variability of Log transformed Pk Parameters Test-T2 Vs Reference-R

\begin{tabular}{|c|c|c|c|}
\hline Measures & $\operatorname{Cmax}(\mathrm{ng} / \mathrm{mL})$ & AUCO-t (ng.h/mL) & AUC0-inf (ng.h/mL) \\
\hline \multicolumn{4}{|c|}{ 90\% Confidence Interval (T2 Vs. R) } \\
\hline ln-transformed Lower & 102.04 & 95.26 & 95.36 \\
\hline Upper & 118.20 & 116.01 & 116.05 \\
\hline Power (\%) & 99.9 & 97.9 & 98.0 \\
\hline Bioequivalence & YES & YES & YES \\
\hline
\end{tabular}

The results of this study demonstrate that the criteria used to assess bioequivalence between the tests (T1 and T2) and reference (R) formulations were fulfilled.

The tests (T1 and T2) to reference (R) ratio of geometric least square means with corresponding 90\% CI for lntransformed pharmacokinetic parameter Cmax, AUC0-t and AUC0- $\infty$ were within the acceptance range of 80.00 $125.00 \%$.

\section{DISCUSSION AND CONCLUSION}

The objective of the present study is to demonstrate bioavailability between Test Products (T1 \& T2) Metoprolol succinate Extended Release Tablets $50 \mathrm{mg}$ and Reference Product (R) TOPROL-XL® (Metoprolol Succinate) Extended Release Tablets $50 \mathrm{mg}$, Under Fasting Condition in Normal, Healthy, Adult, and Male Human Subjects in a randomized crossover study.

The quantification of Metoprolol Succinate in plasma samples is performed in accordance with GLP requirements. The analytical methods LC/MS-MS allow specific and sensitive determination of Metoprolol Succinate in plasma. The calibration ranges are validated for the analysis of plasma samples show linearity between $0.203 \mathrm{ng} / \mathrm{mL}$ to $100.500 \mathrm{ng} / \mathrm{mL}$ for Metoprolol Succinate and the validation parameters of the method fulfilled international requirements for method validation.

The relative bioavailability results indicated that "Test Product-T1 vs. Reference Product-R and Test Product-T2 vs. Reference Product-R" ratio of geometric means for AUC (0t), AUC $(0-\infty)$ and Cmax were $102.6 \%, 103.3 \%$ and $103.5 \%$ for Test Product-T1 vs. Reference Product-R and $109.8 \%$, $105.1 \%$ and $105.2 \%$ respectively, which lie within the bioequivalence range $80.00 \%$ to $125.00 \%$.

The $90 \%$ confidence intervals for $\operatorname{AUC}_{(0-\mathrm{t})}, \mathrm{AUC}_{(0-\infty)}$ and $\mathrm{C}_{\max }$ "Test Product-T1 vs. Reference Product-R and Test ProductT2 vs. Reference Product-R" were $95.63 \%$ to $110.04 \%$, $94.04 \%$ to $113.52 \%$ and $94.19 \%$ to $113.63 \%$ for Test Product-T1 vs. Reference Product-R and $102.04 \%$ to 118.20 $\%, 95.26 \%$ to $116.01 \%$ and $95.36 \%$ to $116.05 \%$ for Test Product-T2 vs. Reference Product-R respectively, which lie within the bioequivalence range $80.00 \%$ to $125.00 \%$.

The results of this study demonstrate that the criteria used to assess bioequivalence between the tests (T1 and T2) and reference (R) formulations were fulfilled.
The tests (T1 and T2) to reference (R) ratio of geometric least square means with corresponding $90 \%$ CI for lntransformed pharmacokinetic parameter Cmax, AUC0-t and AUC0- $\infty$ were within the acceptance range of 80.00 $125.00 \%$.

Therefore, the Test Products (T1 \& T2) are considered to be bioequivalent to the Reference Product- $R$ under fasting condition for Metoprolol.

\section{REFERENCES:}

1. Gupta PK and Robinson JR. Oral controlled release delivery. Treatise on controlled drug delivery., 1992; 93(2):545-555.

2. Jantzen GM and Robinson JR. Sustained and Controlled-Release Drug Delivery systems. Modern Pharmaceutics., 1995; 121(4): 501-502.

3. Wani MS. Controlled Release System A Review; Pharmaceutical Reviews., 2008; 6(1):41-46.

4. Hayashi T. Formulation, study and drug release mechanism of a new Theophylline sustained release preparation, Int. J Pharm., 2005; 304: 91-101.

5. Venkatraman S, Davar $\mathrm{N}$ and Chester A. An overview of controlled release systems: Edited by Donald L Wise, New York, Marcel Dekker Inc. Handbook of Pharmaceutical controlled release Technology., 2000; 431-465.

6. Kulkarni JS., Pawar AP., Shedbakar VP., Biopharmaceutics and Pharmacokinetics, CBS Publishers and Distributors, New Delhi, 2006, page No. 249-262.

7. U.S. Department of Health and Human Services, Guidance for Industry Bioavailability and Bioequivalence Studies for Orally Administered Drug Products - General Considerations, Food and Drug Administration Center for Drug Evaluation and Research (CDER), March 2003 BP, Revision 1.

8. Saudi Food and Drug Authority, Bioequivalence Requirements guidelines, Kingdom of Saudi Arabia, Drug Sector, Draft May 2005.

9. Marzo A, -Clinical pharmacokinetic registration file for NDA and ANDA procedures.|| Pharmacol Res. 1997, 36(6):425-450.

10. Bahar MA, Kamp J, Borgsteede SD, Hak E, Wilffert B: The impact of CYP2D6 mediated drug-drug interaction: a systematic review on a combination of metoprolol and paroxetine/fluoxetine. $\mathrm{Br} J$ Clin Pharmacol. 2018 Dec, page No. 2704-2715.

11. Frishman W., Cheng-Lai A. and Nawarskas J. (2005). Current cardiovascular drugs (4th ed.). Current medicine LLC.

12. Tsigos C, Kyrou I, Kassi E, Chrousos GP. Stress, Endocrine Physiology and Pathophysiology. Endotext. MDText.com, Inc.; 2000.

13. Bahar MA, Kamp J, Borgsteede SD, Hak E, Wilffert B: The impact of CYP2D6 mediated drug-drug interaction: a systematic review on a combination of metoprolol and paroxetine/fluoxetine. $\mathrm{Br} \mathrm{J}$ Clin Pharmacol. 2018 Dec, page No. 2704-2715)

14. University of Illinois-Chicago - Drug Information Group. Metoprolol Tartrate vs. Metoprolol Succinate [Internet]. 2016 [cited 2018 Feb 27]. 